\title{
Estrogen-Related Receptor $\alpha$ Inverse Agonist Enhances Basal Glucose Uptake in Myotubes through Reactive Oxygen Species
}

\author{
Lihua CHEN and Chiwai Wong* \\ Guangzhou Institute of Biomedicine and Health, Chinese Academy of Sciences, Guangzhou International Business \\ Incubator; Guangzhou Science City, Guangzhou 510663, P. R. China. \\ Received December 16, 2008; accepted March 23, 2009
}

Estrogen-related receptors alpha $(\mathrm{ERR} \alpha)$ and peroxisome proliferator-activated receptor $\gamma$ coactivator-1 $\alpha$ (PGC-1 $\alpha$ ) function coordinately to regulate mitochondrial biogenesis and oxidative phosphorylation particularly in muscle tissue. In this study, we addressed the consequences of suppressing the activity of ERR $\alpha$ in L6 myotubes using an ERR $\alpha$ inverse agonist XCT-790. We found that treating differentiated L6 myotubes with XCT-790 reduced the expression of PGC-1 $\alpha$ and suppressed mitochondrial biogenesis. Additionally, XCT-790 increased the production of reactive oxygen species (ROS) which in turn induced the expressions of glucose transporters 1 , 2 , and 5 leading to an increase in glucose uptake and uncoupling protein 3 leading to a reduction of mitochondrial membrane potential. Thus, suppressing the activity of ERR $\alpha$ which is primarily responsible for controlling $\beta$ oxidation would nonetheless indirectly affect glucose uptake in a ROS-dependent manner.

Key words estrogen-related receptor alpha; reactive oxygen species; glucose uptake

Skeletal muscle is one of the key insulin-responsive organs responsible for maintaining normal glucose homeostasis. ${ }^{1)}$ Mitochondrial dysfunction has been closely associated with skeletal muscle insulin resistance in several studies. $^{2-4)}$ In skeletal muscle of human type II diabetics, the expression levels of mitochondrial oxidative phosphorylation (OXPHOS) genes are reduced. ${ }^{5)}$ The OXPHOS genes that are dysregulated in type II diabetic patients are under the transcriptional control of peroxisome proliferator-activated receptor $\gamma$ coactivator- $1 \alpha(\mathrm{PGC}-1 \alpha){ }^{5)}$

Estrogen-related receptors alpha $(\mathrm{ERR} \alpha)$ is an orphan nuclear hormone receptor that displays constitutively active transcriptional activity. ${ }^{6,7)}$ ERR $\alpha$ is expressed in tissues with a high capacity for $\beta$-oxidation of fatty acids including the heart, kidneys, brown adipose tissue and skeletal muscle. ${ }^{8,9)}$ $\operatorname{ERR} \alpha$ is primarily thought to regulate energy homeostasis through interacting with PGC- $1 \alpha$ and coordinately control the transcription of genes in the oxidative phosphorylation pathway. $^{10)}$

Although $\mathrm{ERR} \alpha$ and $\mathrm{PGC}-1 \alpha$ are thought to primarily regulate oxidative phosphorylation and mitochondrial biogenesis, we demonstrated in our previous study that suppressing ERR $\alpha$ activity in adipocytes would nonetheless lead to an increase in glucose uptake primarily due to the production of reactive oxygen species (ROS). ${ }^{11)}$ In the present study, we examined the consequences glucose uptake upon suppressing the activity of ERR $\alpha$ by its inverse agonist XCT-790 in rat L6 myotubes. ${ }^{12,13)}$

\section{MATERIALS AND METHODS}

Reagents Cell culture reagents, including Dulbecco's Modified Eagle's Medium, horse serum and antibiotics/antimycotics were purchased from Gibco (Burlington, ON, Canada), fetal bovine serum was purchased from Hyclone (Burlington, ON, Canada). ${ }^{3} \mathrm{H}-2$-deoxyglucose was purchased from Atom HighTech (Beijing, China). Mn(III) Tetra(4-benzoic acid) porphyrin chloride (MnTBAP) was purchased from Calbiochem (Gibbstown, NJ, U.S.A.). All other chemicals were purchased from Sigma (Oakville, ON, Canada).

Cell Culture and Treatment L6 rat myoblasts were cultivated and differentiated as previously described. ${ }^{14)}$ Differentiation medium was replaced every $48 \mathrm{~h}$ for 6 or $7 \mathrm{~d}$, unless otherwise stated, allowing sufficient time for myotubes formation. All experiments were initiated 6 to 7 days' post-differentiation.

Plasmids Construction To construct the promoter expression vector for PGC- $1 \alpha$, the $2.6 \mathrm{~kb}$ of PGC- $1 \alpha$ promoter was cloned into pGL-3 basic vector by $K p n I$ and $X h o I$ as described, ${ }^{15)}$ the primers were 5'-GTAGGTACCCATTTAAGTTCTCCTGAGAAAAG-3' and 5'-GTACTCGAGTGACGCCAGTCAAGCTTTTT-C-3'. The insert fragments were verified by DNA sequencing (Invitrogen). The pCMX-ERR $\alpha$ plasmid was purchased from FulenGen (FulenGen, China).

Transient Transfection and Luciferase Assays For luciferase assays, differentiated L6 myotubes in 96-well plates were transfected with a pGL3-PGC-1 $\alpha$-promoter luciferase reporter plasmid with or without a $\mathrm{pCMX}-\mathrm{ERR} \alpha$ expression vector using Lipofectamine 2000 according to manufacturer's instructions (Promega). Approximately $8 \mathrm{~h}$ post-transfection, cells were then treated with XCT-790 in various concentrations for $24 \mathrm{~h}$ and luciferase activity was measured and normalized against Renilla luciferase activity as an internal control with a Dual-Luciferase Reporter System (Promega) in a Lumat LB9501 luminometer (Berthold, Wildbad, Germany).

Reactive Oxidant Species (ROS) Assay ROS was measured using a fluorescent dye $2^{\prime}, 7^{\prime}$-dichlorofluorescein diacetate (DCFH-DA) (Beyotime, JiangSu) as previously described. ${ }^{16)}$ In brief, L6 rat myoblasts were differentiated for $7 \mathrm{~d}$ and treated with different concentrations of XCT-790 for the time indicated, and then the myotubes were washed and incubated with $10 \mu \mathrm{M}$ DCFH-DA for $20 \mathrm{~min}$ at $37^{\circ} \mathrm{C}$. Cells were then washed several times and harvested in phosphate buffered saline (PBS). The fluorescence of 2,7-dichlorofluorescein (DCF) was detected with a fluorescence assitsted cell sorter (FACS) calibur (excitation at $488 \mathrm{~nm}$, emission at 
$530 \mathrm{~nm})$ (BD, U.S.A.).

Mitochondrial Mass Mitochondrial mass was measured by loading with MitoTracker green (Molecular Probes, Invitrogen) as previously described. ${ }^{17)}$ Briefly, L6 rat myoblasts were differentiated for $7 \mathrm{~d}$ and treated with different concentrations of XCT-790 for $24 \mathrm{~h}$, and incubated with $150 \mathrm{~nm}$ MitoTracker green for $30 \mathrm{~min}$ at $37^{\circ} \mathrm{C}$ and then fluorescence of separate cells was detected with FACS calibur (excitation at $490 \mathrm{~nm}$, emission at $516 \mathrm{~nm}$ ).

Mitochondrial Membrane Potential The mitochondrial membrane potential was measured by loading with $10 \mu \mathrm{M}$ JC-1 (Beyotime, JiangSu). Monomer JC-1 of green fluorescence forms dimer of red fluorescence when membrane potential is high and when the membrane potential is lower, there is more green fluorescence. L6 rat myoblasts were differentiated for $7 \mathrm{~d}$ and treated with $20 \mu \mathrm{M}$ of XCT-790 for $24 \mathrm{~h}$, and incubated with $10 \mu \mathrm{M} \mathrm{JC}-1$ for $30 \mathrm{~min}$ at $37^{\circ} \mathrm{C}$ in cell culture and fluorescence of separate cells was detected with FACS calibur.

2-Deoxyglucose Uptake Glucose uptake assays were performed in L6 myotubes as previously described. ${ }^{18)} \mathrm{XCT}$ 790 was administered to L6 myotubes 6 days' post-differentiation for $24 \mathrm{~h}$ and cells were washed twice with PBS and then incubated in Kreb's Ringer with $2 \mathrm{~mm}$ pyruvate and $2 \%$ bovine serum albumin (FCB) for $20 \mathrm{~min}$ and another $20 \mathrm{~min}$ in $\mathrm{FCB}$ at $37^{\circ} \mathrm{C}$ with or without $100 \mathrm{~nm}$ insulin. Cells were then maintained in $\mathrm{FCB}$ with the $8.3 \mu \mathrm{Ci} / \mathrm{ml}^{3} \mathrm{H}$-2-deoxyglucose for $10 \mathrm{~min}$. Cells were washed by cold PBS for several times and harvested in the scintillation liquid. Radioactivity was determined by liquid scintillation counting (PE, U.S.A.).

RNA Isolation and Quantitative Real-Time Polymerase Chain Reaction (PCR) TRIzol reagent (Invitrogen) was used to isolate total RNA from L6 myotubes pretreated by drugs according to the manufacturer's instructions. The firststrand cDNA was generated with oligo-d(T) primer by reverse transcription kits (Invitrogen) using $2 \mu \mathrm{g}$ of total RNA. The resulting cDNA was subsequently analyzed with SYBR green kit (Takara, Japan) on an MJ Real-Time PCR System using the $\Delta \Delta C_{\mathrm{t}}$ threshold cycle method. Relative gene expression was normalized to glyceraldehyde-3-phosphate dehydrogenase (GAPDH) or $\beta$-actin levels.

Statistical Analysis Data were analyzed by one way analysis of variance followed by Dunnett's test or two-tailed Student's $t$ test to determine $p$ values according to the number of groups compared. Results are expressed as means \pm S.D. or means \pm S.E.M. for experiments.

\section{RESULTS}

ERR $\alpha$ Inverse Agonist XCT-790 Reduces Mitochondrial Biogenesis in L6 Myotubes Differentiated L6 myotubes have been widely used as an in vitro model system for studying the metabolism of skeletal muscle such as insulininduced glucose uptake, beta-oxidation and glycolysis. ${ }^{19,20)}$ During myoblasts differentiation into myotubes, we found that the mRNA expression levels of $\operatorname{ERR} \alpha$ and PGC- $1 \alpha$ were induced after initiation of differentiation (data not shown). In order to test whether down-regulating the activity of ERR $\alpha$ would alter energy homeostasis, we utilized an ERR $\alpha$ specific inverse agonist XCT-790 to suppress its activity. $^{12,13)}$
The expression of PGC- $1 \alpha$ is partly under the control of ERR $\alpha^{21)}$ We first asked if XCT-790 was effective in reducing the activity of ERR $\alpha$ by checking its regulation on the expression of a luciferase reporter under the control of PGC$1 \alpha$ promoter. Differentiated L6 myotubes were transfected with a PGC- $1 \alpha$ promoter-luciferase reporter with or without an $\operatorname{ERR} \alpha$ expression vector and an internal control Renilla luciferase. Cells were then treated with increasing concentrations of XCT-790 and the luciferase activities were measured $24 \mathrm{~h}$ after compound addition. We found that over-expression of ERR $\alpha$ enhanced the expression of PGC- $1 \alpha$-promoter luciferase reporter and that XCT-790 dose-dependently reduced the ERR $\alpha$-induced regulation (Fig. 1A). Importantly, we found that the endogenous mRNA expression level of PGC-1 $\alpha$ was modestly suppressed by XCT-790 (Fig. 1B). Since PGC- $1 \alpha$ plays an essential role in regulating mitochondrial biogenesis, we then examined the effect of moderately down-regulating the expression level of this regulator on mitochondrial biogenesis. Using a mitochondrial dye Mitotracker Green that stains mitochondrion independent of mitochondrial membrane potential, we found that XCT-790
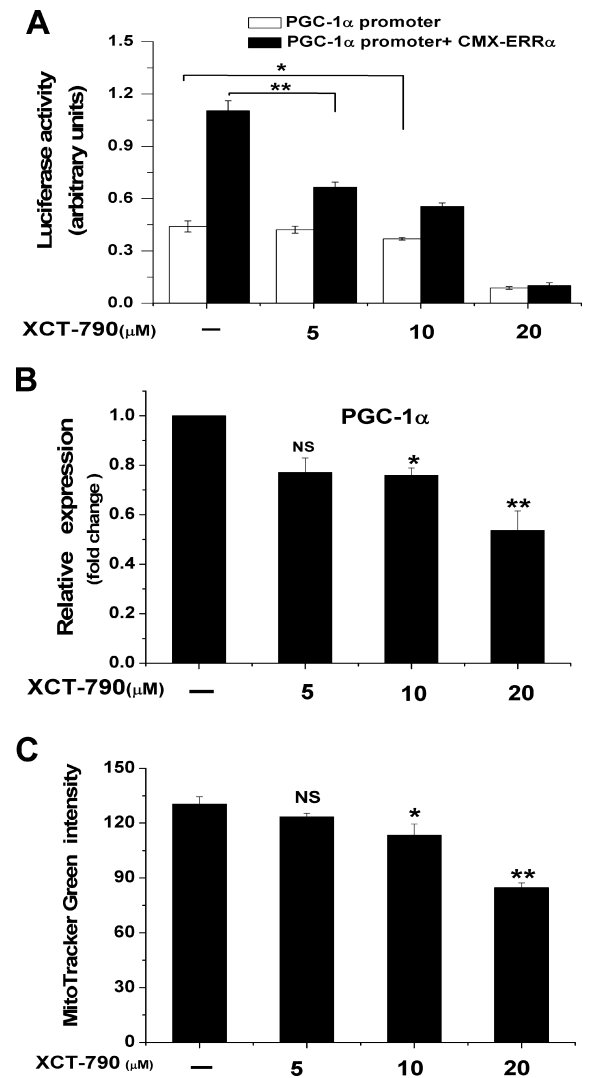

Fig. 1. Effects of XCT-790 on Mitochondrial Biogenesis

(A) Differentiated L6 myotubes were transfected with PGC-1 $\alpha$ promoter-luciferase, PGC- $1 \alpha$ and ERR $\alpha$ expression vectors as described in Materials and Methods. Data are means \pm S.D. from experiments performed in triplicate and are representative of three experiments with similar results. $(n=3 * p=0.031 v s$. dimethyl sulfoxide (DMSO) treated PGC- $1 \alpha$ expression vector transfected cells; $* * p=0.007 v s$. DMSO treated PGC- $1 \alpha$ and ERR $\alpha$ expression vectors co-transfected cells, by Student's $t$ test). (B) mRNA level of PGC-1 $\alpha$ was determined by RT-PCR and normalized with GADPH expression level. The relative expression is presented as the fold of expression level of DMSO treatment. Data are shown as means \pm S.E. of five independent experiments ( $n=5, * p=0.038, * * p=0.0063$, NS: no significance, $v s$. DMSO treated cells, by Dunnett's test). (C) Mitochondrial mass were detected by staining with Mitotracker Green. Data are shown as means \pm S.D. from experiments performed in triplicate and are representative of three other experiments with similar results. $(n=4, * p=0.042, * * p=$ 0.0073 , NS: no significance $v s$. DMSO treated, by Dunnett's test). 
dose-dependently reduced mitochondrial mass (Fig. 1C).

XCT-790 Increases Reactive Oxygen Species Production Mitochondria are sources of cellular reactive oxygen species (ROS). Since ERR $\alpha$ and PGC- $1 \alpha$ coordinately control the transcription of genes in the electron transport chain, reducing the expression of $\mathrm{PGC}-1 \alpha$ may have an impact on ROS production. We examined if XCT-790 treatment would elevate the production of ROS in L6 myotubes. We measured the cellular ROS level by fluorescent dye $2^{\prime}, 7^{\prime}$-dichlorofluorescein diacetate (DCFH-DA) and detected an increase in ROS level upon XCT-790 treatment (Fig. 2A). We next confirmed if the increase in ROS was derived from the mitochondria using an electron transport chain complex I inhibitor rotenone. We found that rotenone blocked the induction of ROS mediated by XCT-790 (Fig. 2B). On the other hand, rotenone did not alter the ability of XCT-790 to suppress mitochondrial biogenesis (Fig. 2C), which strongly suggested that the increase in ROS was derived from the mitochondria as a consequence of XCT-790-mediated alteration of mitochondrial function.

XCT-790 Increases Uncoupling Protein 3 (UCP3) Expression and Lowers Mitochondrial Membrane Potential The accumulation of ROS if left unchecked may inflict deleterious consequences. Therefore, minimizing the production of ROS from the mitochondria is critical to maintain cellular function. We found that the expression levels of anti-oxidative enzymes superoxide dismutase 1 and 2 (SOD1 and SOD2), catalase, and glutathione peroxidase (GPx) were not significantly changed by XCT-790 treatments. On the other hand, we found that the mRNA expression level of uncoupling protein 3 (UCP3) but not uncoupling protein 2 (UCP2) was induced by XCT-790 (Fig. 2D). This XCT-790-mediated induction of UCP3 expression was blocked by an antioxidant $\mathrm{Mn}$ (III) tetra(4-benzoic acid) porphyrin chloride
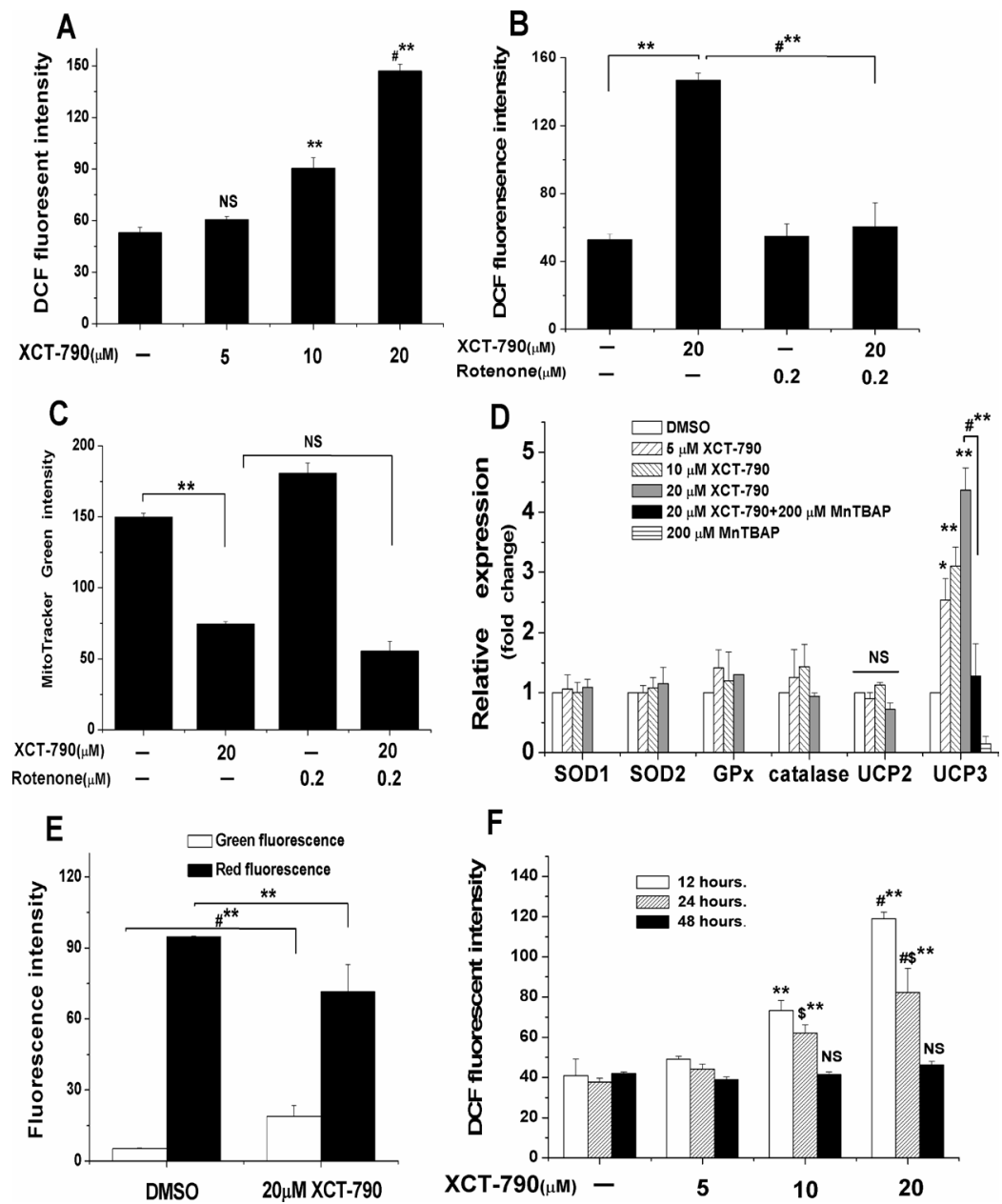

Fig. 2. XCT-790 Decreases Mitochondrial Membrane Potential But Induces ROS Production

(A) Differentiated L6 myotubes were incubated with different concentrations of XCT-790 for $24 \mathrm{~h}$. ROS levels were detected by DCFH-DA probe and analyzed by FACS. Data are presented as means \pm S.D. from experiment performed in triplicate and are representative of four experiments with similar results $(n=4, * * p=0.0044$, \#** $p=0.0031$, NS: no significance $v s$. DMSO treated, by Dunnett's test). (B) Differentiated L6 myotubes were incubated with $20 \mu \mathrm{M}$ XCT- 790 with or without $200 \mathrm{nM}$ rotenone for $24 \mathrm{~h}$. ROS levels were detected as in (A). Data are presented as means \pm S.D. from experiment performed in triplicate and are representative of at least three experiments with similar results. $(n=4$, $* * p=0.0079 v s$. DMSO treated, \#** $p=0.0087 v s$. $20 \mu \mathrm{M}$ XCT-790 treated, by Student's $t$ test). (C) Mitochondrial mass were detected as in Fig. 1C. Data are presented as means + S.D. from experiment performed in triplicate and are representative of at least three experiments with similar results. $(n=4, * * p=0.0081 v s$. DMSO treated, NS: no significance $v s$. $20 \mu \mathrm{M}$ XCT-790 treated, by Student's $t$ test). (D) Differentiated L6 myotubes were incubated different concentrations of XCT-790 with or without $200 \mu \mathrm{M}$ MnTBAP for $24 \mathrm{~h}$. mRNA levels of SOD1, SOD2, GPx, catalase, UCP2 and UCP3 were determined by RT-PCR and normalized with $\beta$-actin expression level. The relative expression is presented as the fold of expression level of DMSO treatment. Data are shown as mean \pm S.E. of at least three independent experiments $(n=5, * p=0.038, * * p=0.0093, \$ * * p=0.0027$ $v s$. DMSO treated, NS: no significance $v s$. DMSO treated, by Dunnett's test; \#** $p=0.0037, v s$. $20 \mu \mathrm{M}$ XCT-790 and $200 \mu \mathrm{M}$ MnTBAP co-treated, by Student's $t$ test). (E) Differentiated L6 myotubes were incubated with $20 \mu \mathrm{M}$ XCT-790 for $24 \mathrm{~h}$. Mitochondrial membrane potential was analyzed by JC-1 fluorescence and the relative amounts of red and green fluorescence were presented compared to DMSO control $(n=3, * p=0.047$, \#* $p=0.043 v s$. DMSO treated, by Student's $t$ test). (F) Differentiated L6 myotubes were incubated with different concentrations of XCT-790 for 12,24 or $48 \mathrm{~h}$. ROS levels were detected as in (A). Data are presented as means \pm S.D. from experiment performed in triplicate and are representative of four experiments with similar results $(n=4, * * p=0.0057$, \#** $p=0.0027, \$ * * p=0.0089$, \#\$** $p=0.0069$, NS: no significance $v s$. DMSO treated, by Dunnett's test). 
(MnTBAP) (Fig. 2D), suggesting that ROS was a primary signal responsible for inducing the expression of UCP3. The increase in UCP3 expression may in turn lower mitochondrial membrane potential $\left(\Delta \Psi_{\mathrm{m}}\right)$ and subsequently ROS production. Using a mitochondrial dye JC-1 that gives a green fluorescence when $\Delta \Psi_{\mathrm{m}}$ is low and a red fluorescence when $\Delta \Psi_{\mathrm{m}}$ is high, we found that $\Delta \Psi_{\mathrm{m}}$ was in fact lowered in cells treated with $20 \mu \mathrm{M}$ XCT-790 (Fig. 2E). Additionally, we found that the XCT-790-mediated increase in ROS was only transient as ROS level declined back to normal level $48 \mathrm{~h}$ after XCT-790 addition, consistent with the decrease in $\Delta \Psi_{\mathrm{m}}$ (Fig. 2F).

XCT-790 Induces Glucose Transporters Expression in Response to an Increase in ROS Production ROS has also been suggested to modulate glucose uptake. ${ }^{22)}$ Since glucose uptake is primarily mediated by glucose transporters in muscle cells, we further investigated if XCT-790 would affect the expression levels of glucose transporters $1-5$ (Glut $1-$ 5). Consistent with Glut-4 as an ERR $\alpha$ target gene, ${ }^{23)}$ we found that the mRNA expression level of Glut-4 but not Glut-3 was modestly reduced by XCT-790 (Fig. 3A). On the other hand, the expression of Glut-1, Glut-2 and Glut-5 were induced by XCT-790 treatment and their inductions were blocked by the anti-oxidant MnTBAP (Fig. 3A), suggesting that these inductions were ROS-mediated. We finally checked the effect of XCT-790 on glucose uptake in L6
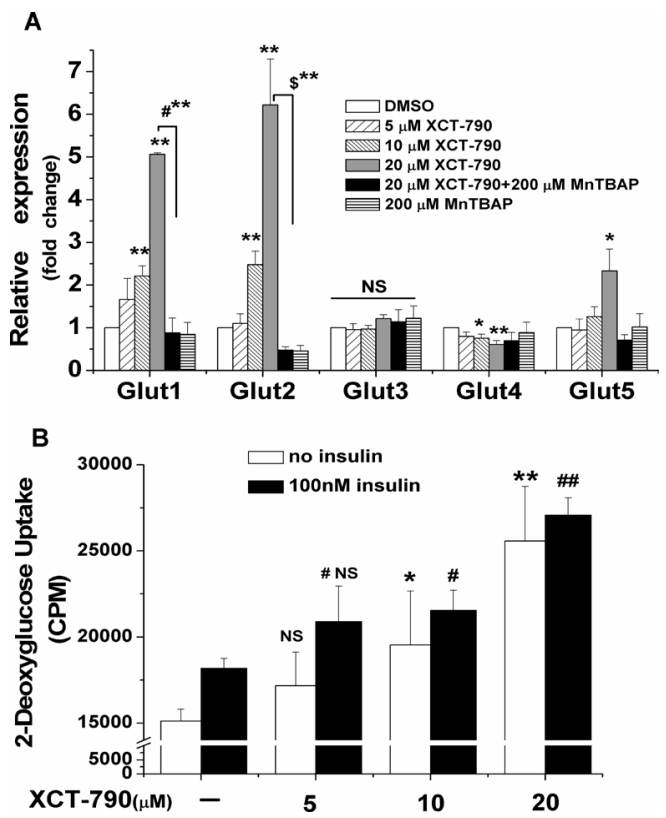

Fig. 3. XCT-790 Induces Glucose Transporters Expressions and Basal Glucose Uptake

(A) Differentiated L6 myotubes were incubated with different concentrations of XCT-790 with or without $200 \mu \mathrm{m}$ MnTBAP for $24 \mathrm{~h}$. mRNA levels of Glut $1-5$ were determined by RT-PCR and normalized with $\beta$-actin expression level. The relative expression is presented as the fold of expression level of DMSO treatment. Data are shown as mean \pm S.E. of at least three independent experiments. $(n=4$, NS: no significance, $* p<0.05$, $* * p<0.01$, vs. DMSO treated, by Dunnett's test $\# * * p=0.0078, \$ * * p=0.003220 \mu \mathrm{M}$ XCT-790 treated $v s .20 \mu \mathrm{M}$ XCT-790 and $200 \mu \mathrm{m}$ MnTBAP co-treated, by Student's $t$ test). (B) Differentiated L6 myotubes were incubated with different concentrations of XCT-790 for $24 \mathrm{~h} .{ }^{3} \mathrm{H}$-deoxyglucose uptake with or without $100 \mathrm{~nm}$ insulin stimulation for $20 \mathrm{~min}$ was measured by liquid scintillation counter (Perkin Elmer). Data are presented as means \pm S.D. from experiment performe in triplicate and are representative of at least three experiments with similar results $(n=3, * p=0.026, * * p=0.0027$, NS: no significance $v s$. DMSO treated without insulin stimulation; \#* $p=0.039$, \#** $p=0.0059$, \#NS: no significance $v s$. DMSO treated with insulin stimulation, by Dunnett's test). myotubes and found that XCT-790 dose-dependently enhanced the basal glucose uptake in the absence of insulin (Fig. 3B), suggesting that suppressing ERR $\alpha$ activity would increase basal glucose uptake through inducing the expressions of glucose transporters. On the other hand, XCT-790 partially blunted the insulin-mediated moderate increase in glucose uptake (Fig. 3B).

\section{DISCUSSION}

ERR $\alpha$ and PGC- $1 \alpha$ corporately control the expression of genes in the electron transport chain, alteration of ERR $\alpha$ and PGC- $1 \alpha$ activities may reduce the functional capacity of the mitochondrion leading to an excess of electron to be transferred to oxygen thus generating ROS. In this study, we indeed found that suppressing the activity of ERR $\alpha$ in muscle cells would lead to a transient increase in ROS which in turn alter the expression of glucose transporters. Previously, we demonstrated in adipocytes that suppressing ERR $\alpha$ activity would also increase glucose uptake in an ROS-dependent manner. ${ }^{11)}$ Although the overall effects are similar, in adipocytes ERR $\alpha$ inverse agonist XCT-790 increases $\Delta \Psi_{\mathrm{m}}$; whereas, in muscle cells XCT-790 reduces $\Delta \Psi_{\mathrm{m}}$. The primary difference is likely due to the muscle specific induction of UCP3 by ROS (Fig. 2D). We did not observe any changes in UCP3 expression in adipocytes treated with XCT-790 (data not shown). This cell type specific regulation of UCP3 by ROS is a subject of continued investigation.

While XCT-790 appears to regulate the expression of uncoupling proteins in a cell-type dependent manner, it suppresses the expression of Glut-4 in adipocytes as we previously demonstrated and muscle cells in this current study. Glut-4 is regulated not only by ERR $\alpha,{ }^{23)}$ but also by liver X receptor $\beta(\operatorname{LXR} \beta){ }^{24)}$ PGC-1 $\alpha$ can serve as a coactivator for both ERR $\alpha$ and LXR $\beta$. By reducing the expression level of PGC- $1 \alpha$, XCT-790 likely suppresses the expression of Glut-4 through reducing the amount of coactivator being recruited to Glut-4 promoter via $\operatorname{ERR} \alpha$ or $\operatorname{LXR} \beta$. On the other hand, Glut-1, Glut- 2 and Glut- 5 are induced by XCT-790 indirectly through an ROS-dependent manner. Interestingly, ROS derived from the mitochondria has been shown to activate hypoxia-inducible factor $\alpha$ (HIF-1 $\alpha$ ) and HIF- $1 \alpha$ can stimulate the expression of Glut-1. ${ }^{25,26)}$ It is therefore reasonable to speculate that XCT-790 alters mitochondrial function, induces ROS, activates HIF- $1 \alpha$ and stimulates the expressions of glucose transporters.

Chronic inflammation is associated with many metabolic diseases including diabetes. Cytokines like tumor necrosis factor alpha (TNF $\alpha$ ) and interleukin-6 (IL-6), which are elevated during inflammatory responses, are thought to contribute to insulin resistance. ${ }^{27)}$ These inflammatory cytokines can induce the production of ROS which activates stress responsive kinases like c-Jun N-teriminal kinase $(\mathrm{JNK}){ }^{28)} \mathrm{JNK}$ can in turn reduce insulin sensitivity by phosphorylating insulin receptor substrates and reducing their abilities to interact with downstream effectors. ${ }^{29)}$ Our discovery that ERR $\alpha$ inverse agonist XCT-790 alters mitochondrial function and induces ROS raises the possibility that reduced insulin sensitivity may also be a result of suppressed ERR $\alpha$ activity. Further investigation into this pathway should shed light into how OXPHOS genes that are dysregulated in type II diabetic 
patients may be a contributing factor to reduced insulin sensitivity.

Acknowledgements We sincerely thank Dr. Brian Lavan for providing the L6 muscle cells. The research is supported by Grants from the National Natural Science Foundation of China \#30672463, the National Basic Research Program of China (973-Program) \#2006CB50390, and the Knowledge Innovation Program of the Chinese Academy of Sciences \# KSCX2-YW-R-085.

\section{REFERENCES}

1) Petersen K. F., Befroy D., Dufour S., Dziura J., Ariyan C., Rothman D. L., DiPietro L., Cline G. W., Shulman G. I., Science, 300, 1140-1142 (2003).

2) Kelley D. E., He J., Menshikova E. V., Ritov V. B., Diabetes, 51, 2944-2950 (2002).

3) Petersen K. F., Dufour S., Befroy D., Garcia R., Shulman G. I., $N$. Engl. J. Med., 350, $664-671$ (2004).

4) Simoneau J.-A., Kelley D. E., J. Appl. Physiol., 83, 166-171 (1997).

5) Wu Z., Puigserver P., Andersson U., Zhang C., Adelmant G., Mootha V., Troy A., Cinti S., Lowell B., Scarpulla R. C., Spiegelman B. M., Cell, 98, 115-124 (1999).

6) Giguere V., Trends Endocrinol. Metab., 13, 220-225 (2002).

7) Horard B., Vanacker J. M., J. Mol. Endocrinol., 31, 349-357 (2003).

8) Giguere V., Yang N., Segui P., Evans R. M., Nature (London), 331, 91-94 (1988)

9) Ichida M., Nemoto S., Finkel T., J. Biol. Chem., 277, 50991—50995 (2002).

10) Huss J. M., Torra I. P., Staels B., Giguere V., Kelly D. P., Mol. Cell. Biol., 24, 9079-9091 (2004).

11) Nie Y. H., Wong C. W., J. Cell. Mol. Med., 9999 (2008), accepted article.

12) Mootha V. K., Handschin C., Arlow D., Xie X., St. Pierre J., Sihag S., Yang W., Altshuler D., Puigserver P., Patterson N., Willy P. J., Schulman I. G., Heyman R. A., Lander E. S., Spiegelman B. M., Proc. Natl.
Acad. Sci. U.S.A., 101, 6570-6575 (2004).

13) Busch B. B., Stevens W. C., Martin R., Ordentlich P., Zhou S., Sapp D. W., Horlick R. A., Mohan R., J. Med. Chem., 47, 5593-5596 (2004).

14) Lawson M. A., Purslow P. P., Cells Tissues Organs, 167, 130-137 (2000).

15) Handschin C., Rhee J., Lin J., Tarr P. T., Spiegelman B. M., Proc. Natl. Acad. Sci. U.S.A., 100, 7111-7116 (2003).

16) LeBel C. P., Ischiropoulos H., Bondy S. C., Chem. Res. Toxicol., 5, 227-231 (1992).

17) Civitarese A. E., Carling S., Heilbronn L. K., Hulver M. H., Ukropcova B., Deutsch W. A., Smith S. R., Ravussin E., PLoS Med., 4, e76 (2007).

18) Somwar R., Sweeney G., Ramlal T., Klip A., Clin. Ther., 20, 125-140 (1998).

19) Yonemitsu S., Nishimura H., Shintani M., Inoue R., Yamamoto Y., Masuzaki H., Ogawa Y., Hosoda K., Inoue G., Hayashi T., Nakao K., Diabetes, 50, 1093-1101 (2001).

20) Perdomo G., Commerford S. R., Richard A.-M. T., Adams S. H., Corkey B. E., O'Doherty R. M., Brown N. F., J. Biol. Chem., M403566200 (2004).

21) Arany Z., Lebrasseur N., Morris C., Smith E., Yang W., Ma Y., Chin S., Spiegelman B. M., Cell Metabolism, 5, 35-46 (2007).

22) Katz A., J. Appl. Physiol., 102, 1671-1676 (2007).

23) Dufour C. R., Wilson B. J., Huss J. M., Kelly D. P., Alaynick W. A., Downes M., Evans R. M., Blanchette M., Giguere V., Cell Metabolism, 5, 345-356 (2007).

24) Laffitte B. A., Chao L. C., Li J., Walczak R., Hummasti S., Joseph S. B., Castrillo A., Wilpitz D. C., Mangelsdorf D. J., Collins J. L., Saez E., Tontonoz P., Proc. Natl. Acad. Sci. U.S.A., 100, 5419-5424 (2003).

25) Chandel N. S., Maltepe E., Goldwasser E., Mathieu C. E., Simon M. C., Schumacker P. T., Proc. Natl. Acad. Sci. U.S.A., 95, 11715-11720 (1998).

26) Chen C., Pore N., Behrooz A., Ismail-Beigi F., Maity A., J. Biol. Chem., 276, 9519-9525 (2001).

27) Hotamisligil G. S., Peraldi P., Budavari A., Ellis R., White M. F., Spiegelman B. M., Science, 271, 665-670 (1996).

28) Jennifer L. M., Nikki J. H., J. Cell. Physiol., 192, 1-15 (2002).

29) Aguirre V., Uchida T., Yenush L., Davis R., White M. F., J. Biol. Chem., 275, 9047-9054 (2000). 